\title{
Rheumatology education in India: a survey-based cross sectional study
}

\author{
Latika Gupta ${ }^{1}$ ([) Hiba Khan ${ }^{2} \cdot$ Mehul Sinha $^{3} \cdot$ Durga P. Misra $^{1} \cdot$ Rajat Kharbanda ${ }^{1} \cdot$ Tulika Chatterjee $^{4} \cdot$ Arvind Nune $^{5}$. \\ Vikas Agarwal ${ }^{1}$ (D)
}

Received: 17 June 2021 / Accepted: 27 July 2021 / Published online: 6 August 2021

(c) The Author(s), under exclusive licence to Springer-Verlag GmbH Germany, part of Springer Nature 2021

\begin{abstract}
This study aimed at understanding the perception and perspectives of rheumatology trainees about specialist training in India. Rheumatology trainees (Doctorate of Medicine, Diplomate of National Board) in Indian universities (2010 onwards) were contacted to complete a validated e-survey consisting of 41 questions to evaluate the current rheumatology training in India. Of 53 respondents (M:F 3.4:1, mean age 37 years \pm 12.7 ), 81.1\% trained at government hospitals, and $15.1 \%$ trained at private hospitals. During training period, $37.5 \%$ respondents were exposed to $6-7 \mathrm{~h}$ of didactics/week. They treated nearly 175 patients $(175 \pm 35.4)$ per week and reported a reasonable level of independence in management of patients with common rheumatic diseases (RDs) during their training (7.5 $\pm 0.7 \mathrm{SD})$. However, nearly one-third of the trainees were not exposed to basic immunology and laboratory techniques. Similarly, placement in the radiology department was not a part of the curriculum for nearly half of the trainees, $80 \%$ were not confident to manage paediatric RDs and soft tissue rheumatism. Almost 60\% did not feel comfortable in addressing ancillary care including patient counselling as they had not received formal training. Among the participants, 59\% were not satisfied by the current system of assessment, $86.8 \%$ suggested for multiple time point-based assessment systems and $45.3 \%$ preferred objective and subjective assessment in final examinations. Rheumatology training in India offers notable exposure to patients and independence in managing cases. However, there is an unmet need for improvement in training in the field of laboratory, radiology and ancillary care, and to overhaul assessment system by including objective evaluation.
\end{abstract}

Keywords Rheumatology $\cdot$ Surveys $\cdot$ Rheumatic diseases $\cdot$ Perception $\cdot$ India

\section{Introduction}

Latika Gupta

drlatikagupta@gmail.com

Durga P. Misra

durgapmisra@gmail.com

1 Department of Clinical Immunology and Rheumatology, Sanjay Gandhi Postgraduate Institute of Medical Sciences, Lucknow, India

2 Dubai Medical College, Dubai, United Arab Emirates

3 Kasturba Medical College, Mangalore, Karnataka, India

4 College of Medicine, University of Illinois At Chicago, Illinois, USA

5 Southport and Ormskirk NHS Trust, Southport, UK

Rheumatic disorders are common in the general population. Despite a global burden of rheumatic diseases (RDs), rheumatology in India is still in infancy. With an estimated population of 1.2 billion, of which up to $24 \%$ may suffer from rheumatic disorders annually, less than 1500 rheumatologists cater to a burden of chronic illnesses, amounting to a dismal doctor-patient ratio [1,2]. Twenty-six physicians are trained in this sub-speciality each year, amounting to less than 400 rheumatologists ever enrolled in a structured training programme, a miniscule number to tackle the evergrowing needs of the population $[1,2]$. The number of colleges offering structured speciality courses is limited. With the ever-growing population, there is a need to improve the current doctor-patient ratio through various means. While incorporating rheumatology education into the undergraduate and postgraduate medical curriculum has been examined 
previously, there is little investigation into the quality and structure of rheumatology training as a specialist in India. Understanding the current status of the structure of rheumatology training in India will help us gather the lacunae and provide a direction for improvement needed at the grassroots level for better training. Improved training may be the first step in creating a generation of rheumatologists who would further train students in the future.

The structure of rheumatology training in India entails successful completion of a postgraduate degree: doctor of medicine (MD) or Diploma of National Board (DNB) examination in general medicine or paediatrics as a criterion before enrolling in super-specialization degree for rheumatology, better known as Doctorate of Medicine (DM) [3]. On a broader perspective, rheumatology training is a culmination of a deeper understanding of clinical subjects such as medicine, basic sciences such as anatomy and physiology and the pathological and immunological basis of the diseases and laboratory sciences. In Asia Pacific League of Associations for Rheumatology (APLAR) countries, where physicians practice in a resource constraint setting with high patient demand, the physicians must learn and adapt clinical skills to manage a massive workload which consist of patient-related activity, teaching and administrative work. However, many challenges are faced, such as limited access to ancillary healthcare support staff such as nurse specialists, counsellors, physiotherapists, occupational therapists and nutritionists. Besides this, the trainees need to be aware of research methodologies and publication ethics in order to conduct relevant research work. Another important yet overlooked aspect of rheumatology training is research, which is essential in order to keep up to date with rheumatology advancements and effectively treat patients.

In India, Rheumatology training is still an emerging speciality with very few sub-specialities across the country. A review suggests that a cost-restrained environment requires cost-effective solutions in the healthcare practice to improve patient care [4]. They also recommended to update existing Indian guidelines and develop new guidelines for the management of rheumatic diseases in India using up-to-date methodology. On the other hand, with the novel COVID19 pandemic exacerbating trainings by a magnitude, these solutions have been disrupted. As training programs, clinical directors and educators implement new teaching strategies to conform to the social distancing rules, telemedicine integrated training was the ultimate hope for the future [5]. Technology has become the international remedy to the halt in all education systems during the pandemic and may also be a solution to all the previous impediments that existed before the pandemic.

In this study we aimed to understand the deficiencies in the current rheumatology education in India. We conducted an email survey among the trainees to understand their perceptions and perspectives on rheumatology training and changes required to improve it.

\section{Methods}

Rheumatology trainees (DM, DNB) who were enrolled in rheumatology training programme in the Indian universities from 2010 onwards were contacted over email to complete a validated e-survey designed on an online cloud-based website (Survey Monkey®.com). A total of 225 questionnaires were distributed and 53 of them responded with a completed survey. The intent was to cover various aspects of rheumatology training in India and identify the changes that need to be introduced to improve their training.

\section{Survey design}

The cover letter included an introduction to the team conducting the survey, intent of the survey, consent for participation and publication and data anonymization process. It was circulated on 24 February 2020, and multiple periodic reminders were sent over 5 months. Finally, the surveyed group was sent one additional reminder on WhatsApp portal to complete the survey. The survey responses were allowed until 22 July 2020.

\section{Survey tool}

The anonymized e-survey consisted of 41 questions. The majority were multiple-choice questions with varying number of options and ten questions were matrix-based involving a score-based opinion. Open-ended choices were provided where deemed necessary. The questionnaire was designed to assess respondent characteristics (6 questions), the structure of rheumatology training (11 questions), skill relevance in outcome-based measurement (6 questions), research involvement ( 9 questions), the role of institutes ( 2 questions), student's perspective of methods of assessment currently employed in rheumatology training (3 questions), the role of continued medical education (CME) (3 questions). All questions required a mandatory close-ended response, except opinion-based questions to improve rheumatology training in India.

An independent rheumatology panel comprising three rheumatology consultants and two fellows-in-training reviewed the questions to check and confirm the content validity of the survey [6]. The first draft of the survey was prepared following consultation with the team. The questionnaire was scrutinised for grammatical errors, lacking clarity of idea, relevance and suitability of possible answers and content validity. The questionnaire was then piloted (for completion of the survey) with three rheumatology fellows 
(who were not included in the survey development) on two occasions 4 days apart to assess the test-retest validity [7].

\section{Sampling strategy}

The questionnaire was circulated among rheumatology trainees in India via email and were informed of its purpose to be published in a peer-reviewed journal. Informed consent was obtained, and no incentives were offered for completing the survey.

\section{Data handling and confidentiality}

Data collection was partly anonymous, except the internet protocol (IP) addresses and email lists remaining with the first and corresponding author. Other authors had access to the synthesized data in tables without linked identifiers. Exemption from review had been obtained from the Institutional Ethics Committee (2018-62-IP-EXP) of SGPGIMS, Lucknow, since this was a survey of educational practices, as per local guidelines. It was dated on 20-Apr-2018.

\section{Statistical analysis}

We used descriptive statistics and figures designed using SurveyMonkey. We presented data using median (SD/IQR), percentage and standard deviation. We used a numerical scale $0-10$ to indicate the strength of participants' opinion of the participants based on scale guide (0-1: Strongly negative, 2-4: Borderline, 5-7: Positive, 8-10: Strong positive).

\section{Results}

\section{Respondent characteristics}

Out of the 53 respondents, (M:F ratio- 3.4:1, 37 years \pm 12.7 ) $47(98.1 \%)$ had completed post-graduation in Medicine. Fifty-eight per cent of the individuals were early-career physicians, within 2 years or less since completion of rheumatology training. Most of the respondents (81.1\%) had completed their training from government hospitals, all being in India. Around $40 \%$ were academics, and $28.3 \%$ were involved in both academics and private practice. Forty-three per cent were engaged in academic activities at least $10 \mathrm{~h}$ per week. (Table 1).

\section{Structure of rheumatology training}

During the training period, almost one-third of the respondents $(37.5 \%)$ were exposed to 6-7hourss of didactics on an average weekly, while $26.7 \%$ of the participants received $8-10 \mathrm{~h}$ of bedside/ward round discussion per week. The
Table 1 Respondent Characteristics

\begin{tabular}{|c|c|}
\hline Number of respondents & 53 \\
\hline Median age (IQR) & 37 \\
\hline \multicolumn{2}{|l|}{ Gender } \\
\hline Male & $41(77.4 \%)$ \\
\hline Female & $12(22.6 \%)$ \\
\hline \multicolumn{2}{|l|}{ Qualification } \\
\hline \multicolumn{2}{|c|}{ Post-graduation in Medicine } \\
\hline MD & $47(88.7 \%)$ \\
\hline DNB & $5(9.4 \%)$ \\
\hline \multicolumn{2}{|c|}{ Post-graduation in Paediatrics } \\
\hline MD & $1(1.9 \%)$ \\
\hline DNB & 0 \\
\hline \multicolumn{2}{|c|}{$\begin{array}{l}\text { Years of practice since completion of rheumatology } \\
\text { training }\end{array}$} \\
\hline 2 and less & $31(58.5 \%)$ \\
\hline 3 to 5 & $17(32.1 \%)$ \\
\hline 6 and more & $5(9.4 \%)$ \\
\hline \multicolumn{2}{|l|}{ Training institute } \\
\hline Government hospital & $43(81.1 \%)$ \\
\hline Private hospital & $8(15.1 \%)$ \\
\hline Other & $2(3.8 \%)$ \\
\hline \multicolumn{2}{|l|}{ Countries of training } \\
\hline India & $53(100 \%)$ \\
\hline UK & $2(3.8 \%)$ \\
\hline \multicolumn{2}{|c|}{$\begin{array}{l}\text { Time dedicated to academics after compleing training } \\
\text { (in hours/week) }\end{array}$} \\
\hline Less than 10 & $23(43.4 \%)$ \\
\hline 10 to 30 & $19(35.9 \%)$ \\
\hline 31 to 60 & $9(16.9 \%)$ \\
\hline More than 61 & $2(3.8 \%)$ \\
\hline \multicolumn{2}{|l|}{ Job profile } \\
\hline Academics & $23(43.4 \%)$ \\
\hline Private practice & $12(22.6 \%)$ \\
\hline Both & $15(28.3 \%)$ \\
\hline Trainees & $3(5.6 \%)$ \\
\hline
\end{tabular}

classroom teaching consisted largely of journal clubs (100\%), case presentations $(94.3 \%)$, guidelines review (86.8\%), outcome measures in RDs $(86.8 \%)$ and basic Immunology (83\%). Multidisciplinary team meets (MDT), allergies, applied immunology and clinical audits to improve patient care were neglected aspects of classroom teaching (Fig. 1A).

A two-thirds of the participants reported that applied immunology, radiology, pathology and other speciality MDT meet, audits of patient care, literature scans, clinicopathologic conference and outcome measures in RDs (5-10) benefitted them for delivering patient care. (Fig. 1A, B).

Training in laboratory techniques was low across centres, with nearly one-third not been exposed to basic 

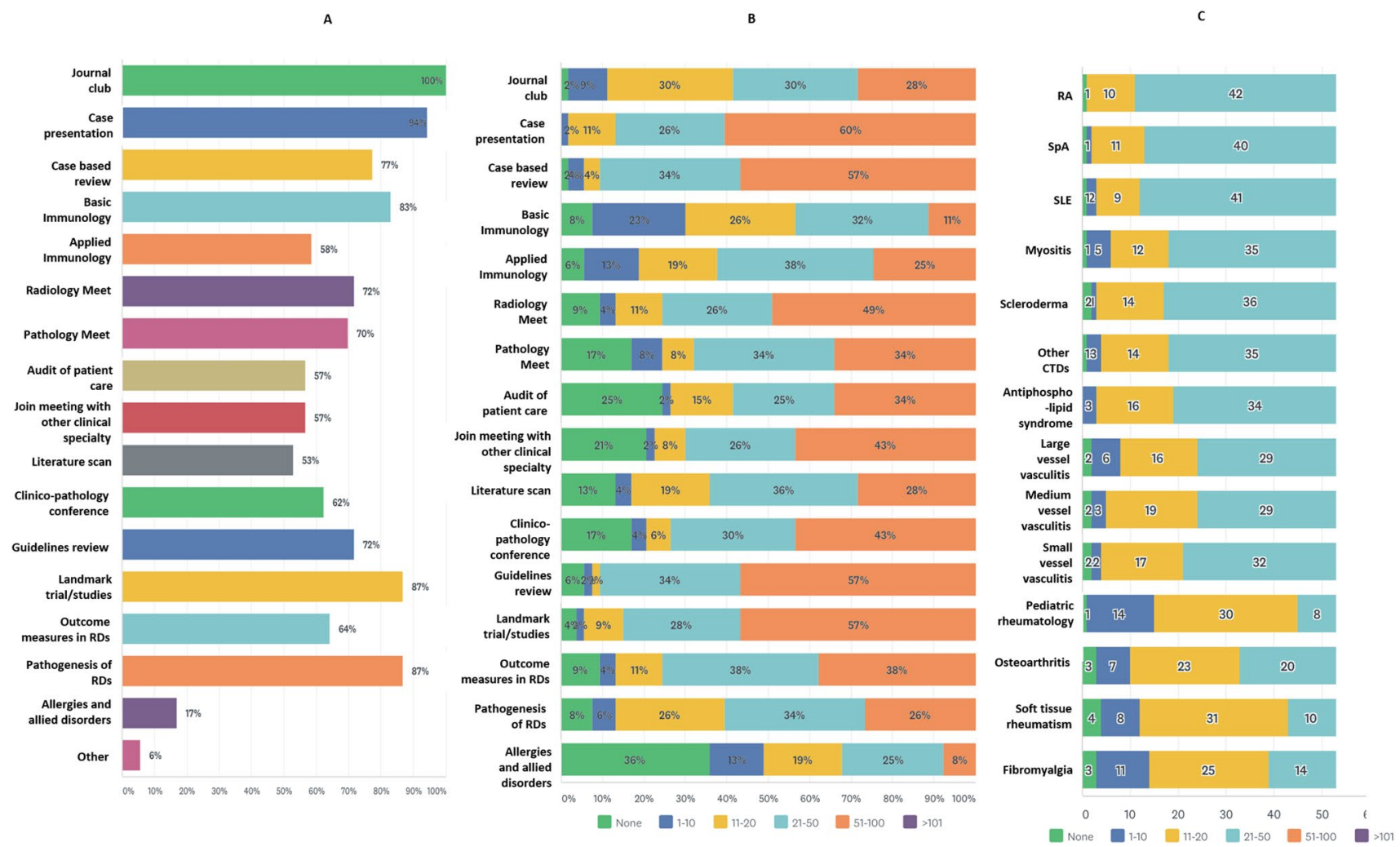

Fig. 1 A What all was the classroom teaching composed of? B What is your perceived level of benefit towards delivering patient care from each of these? C On a scale of 0-10, how confident do you feel in delivering patient care in each of these diseases?

immunology techniques. Similarly, radiology postings were not a part of the curriculum for nearly half, consisting of observing scans with radiologist (46.2\%), workup $(49.1 \%)$ and regular musculo-skeletal USG posting $(60.4 \%)$. One-third (35.8\%) were not exposed to the management of paediatric rheumatology diseases during the course.

\section{Skill base relevant to procedures and outcome measure- based assessment}

The participants reported achieving a reasonable level of independence in management of patients with RD during their training $(7.5 \pm 0.7)$. They treated, on an average, 175 patients $(175 \pm 35.35)$ per week during their training. Majority of respondents ( $>60 \%)$ felt very confident in delivering patient care in rheumatoid arthritis, spondyloarthropathy, systemic lupus erythematosus, myositis, scleroderma, vasculitis and antiphospholipid syndrome. Notably, more than $80 \%$ of participants were not confident enough to manage paediatric patients with RD and soft tissue rheumatism (Fig. 1C).

\section{Procedural skills}

Trainees in general were satisfied with their training in procedural skills. A majority (70\%) of trainees administered over 100 intra-articular injections on an average during their training. Most felt reasonably confident (10) with respect to intra-articular injections (Fig. 2B).

However, almost one-third had never carried out a skin biopsy and 1 in 5 individuals had not conducted a sonographically guided procedure. Almost half of the respondents confirmed not undertaking a nerve biopsy (54\%), a muscle biopsy (45\%) or a kidney biopsy (49\%).

\section{Outcome measures in RD}

Nearly one-third were not accustomed to recording outcome measures in RDs (35.85\%) during training.

\section{Ancillary care}

Notably, almost $60 \%$ of practitioners did not feel comfortable in addressing cardiovascular risk assessment, mental health issues, bone health, physiotherapy, rehabilitation, 


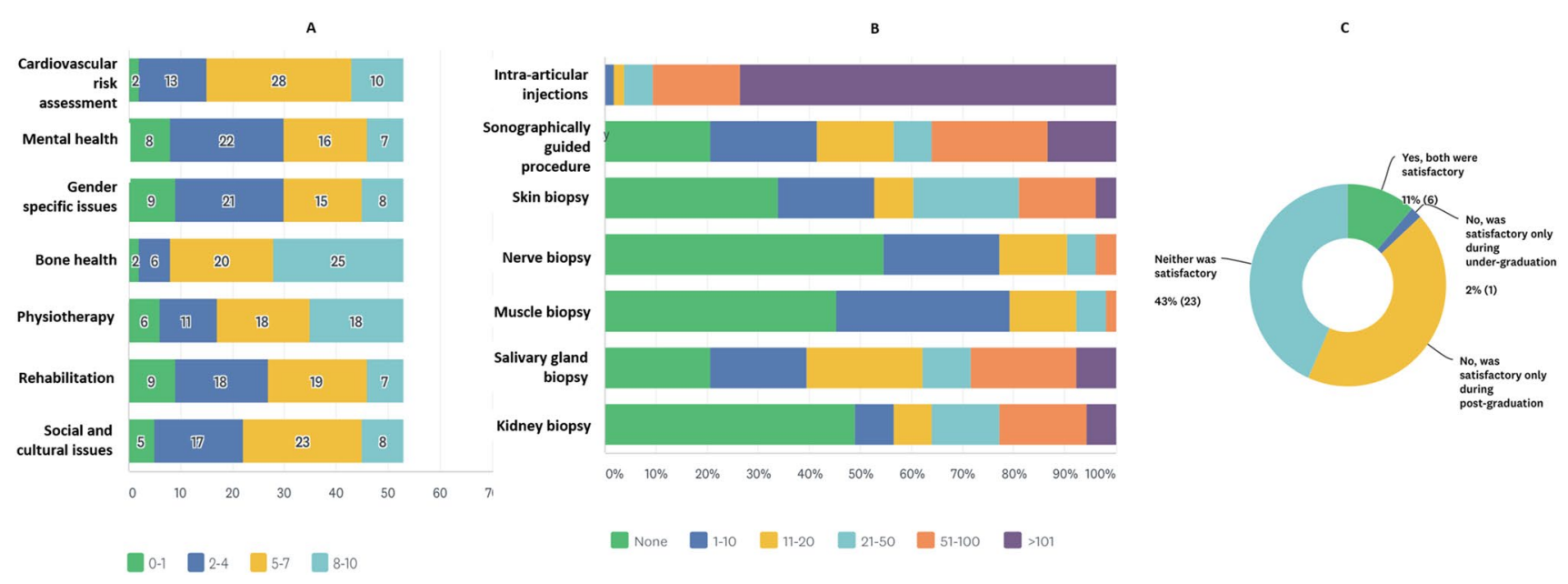

Fig. 2 A How often did/do address these co-morbidities and issues by yourself in a patient with rheumatic disease because allied services were/are not available? B How many procedures (on an average) did

vaccination and patient counselling as they had not received formal training (Supplementary Table, Fig. 2A).

Almost one-third of the participants did not deem themselves fit to manage social, cultural issues and gender-specific issues in a patient with RD. $43 \%$ of trainees picked the scale of 5-7 for the frequency of facing social and cultural issues in a patient. Despite feeling incompetent to handle these issues, they had to address these in the clinic as allied service personnel such as nurses, physiotherapists and counsellors were not available (Supplementary Table).

\section{Research skill development}

As part of their rheumatology training thesis, most of the respondents $(75.5 \%)$ conducted a clinical cohort or case control studies. However, clinical trials (26\%), lab-based study (32\%) and retrospective record review-based studies (26\%) were less commonly preferred during training. More than $60 \%$ of the participants had not written at least 1-2 case reports or original articles during training. Moreover, half of them had not written a review article, and a majority (94\%) had never conducted a meta-analysis. Notably, most respondents felt that thesis-related research work positively impacted $(5.5 \pm 2.1)$ their perspective towards patient care (Table 2).

However, familiarity with publication ethics was low, and $64.1 \%$ had not attended a structured course; $67.9 \%$ were not confident based on self-reading; a large majority being neither $(45.3 \%)$ familiar nor confident.

Similarly, familiarity with statistical methods of research was dismal, with nearly two-thirds having not attended a structured course, and four in five $(79.2 \%)$ being unconfident based on self-reading. A majority (52.8\%) were familiar but you do during training? C Was the rheumatology training received by you during under graduation/post-graduation adequate?

not confident. Most of the respondents felt the need for epidemiology (54\%) and statistics (71\%) to be included in the training curriculum.

Notably, the participants found the time allotted to them exclusively for research adequate $(6 \pm 5.6)$. The respondents unanimously agreed $(10 \pm 0)$ that inclusion of research should be compulsory in rheumatology training curriculum. Proposals to further boost research initiatives during the training included exclusive time apart from regular clinical and academic duties (79.25\%), dedicated funds $(60.3 \%)$, support staff (56.6\%), having better academic opportunities for a career $(39.6 \%)$ and availability of relevant topics for research $(35.8 \%$, Table 3$)$.

\section{Collaborative teaching and exchange programmes}

Nearly one-third respondents perceived that rotation at another institution for rheumatology training would definitely improve the skill-base towards better patient care.

\section{Rheumatology training in undergraduate and postgraduate curricula}

A significant proportion of the respondents (43.4\%) found rheumatology training inadequate at both undergraduate (MBBS) and postgraduate (MD) level. (Fig. 2C, Table 3).

\section{Student assessment}

A majority (59\%) were not satisfied with the current system of assessment. The suggested changes included multiple time point-based assessment systems (monthly or fortnightly) (86.8\%), inclusion of procedural skills in the exam 
Table 2 Training program curriculum

\begin{tabular}{|c|c|}
\hline \multicolumn{2}{|l|}{ Average classroom teaching hour/week } \\
\hline $1-2$ & $6(11.32 \%)$ \\
\hline $3-5$ & $9(16.98 \%)$ \\
\hline $6-7$ & $20(37.74 \%)$ \\
\hline $8-10$ & $15(28.30 \%)$ \\
\hline $11-15$ & $3(5.66 \%)$ \\
\hline$>16$ & 0 \\
\hline \multicolumn{2}{|l|}{ Average bedside teaching hours/week } \\
\hline $1-2$ & $5(9.43 \%)$ \\
\hline $3-5$ & $5(9.43 \%)$ \\
\hline $6-7$ & $14(26.42 \%)$ \\
\hline $8-10$ & $14(26.42 \%)$ \\
\hline $11-15$ & $9(16.98 \%)$ \\
\hline$>16$ & $6(11.32 \%)$ \\
\hline \multicolumn{2}{|l|}{ Were you enrolled in structured laboratory training curriculum? } \\
\hline Yes, online course & $4(7.5 \%)$ \\
\hline Yes, workshop & $28(52.8 \%)$ \\
\hline Laboratory posting in own department & $35(66 \%)$ \\
\hline Laboratory posting in another department & $20(37.7 \%)$ \\
\hline Observed procedures with someone & $15(28.3)$ \\
\hline None of the above & $1(1.8 \%)$ \\
\hline Other (please specify) & $1(1.8 \%)$ \\
\hline \multicolumn{2}{|l|}{ Were you enrolled in a structured radiology curriculum? } \\
\hline Yes, online course & $15(28.3 \%)$ \\
\hline Yes, workshop & $27(50.9 \%)$ \\
\hline Yes, regular ultrasound posting & $21(39.6 \%)$ \\
\hline Yes, observed scans with radiologists & $28(52.8 \%)$ \\
\hline None of the above & $5(9.4 \%)$ \\
\hline Other (please specify) & $1(1.9 \%)$ \\
\hline \multicolumn{2}{|l|}{ Were you enrolled in structured paediatric rheumatology curriculum? } \\
\hline Yes, Online course & $5(9.4 \%)$ \\
\hline Yes, Workshop & $9(16.9 \%)$ \\
\hline Yes, regular management of patients at clinic & $34(64.2 \%)$ \\
\hline None of the above & $13(24.5 \%)$ \\
\hline Other (please specify) & $6(11.3)$ \\
\hline Independence given in patient management on a scale $0-10$ (median IQR) & $7.5(6-9)$ \\
\hline Average number of patients seen in a week (median IQR)? & $175(103-157)$ \\
\hline On a scale of $0-10$, how confident in giving intra-articular injections? & $10(8-10)$ \\
\hline
\end{tabular}

(54.7\%) and an inclusion of feedback from paramedic staff and peers $(37.7 \%)$.

A suggestion to modify the format of theory examinations to combine objective and subjective assessment was made by nearly half of the respondents (45.3\%). A large proportion (79.2\%) felt that regular CME and workshops on general rheumatology would enhance rheumatology practice. Almost everyone agreed $(96 \%)$ that conducting regular $\mathrm{CME} /$ workshops on specific RDs will help them in their rheumatology practice. Almost 4 out of 5 participants had attended at least one international conference/CME annually during training (Table 4).

\section{Discussion}

This survey provides an insight into the quality of the current rheumatology training programme in India and highlights the challenges they face to become independent and wellrounded rheumatologists. During the training period, onethird of respondents were exposed to robust academics of 6-7 h didactics/week, and they treated ginormous volumes cases inclusive of the complete profile of rheumatic diseases. Importantly, most of them reported a reasonable level of independence in management of patients with common RD during their training. However, there seems to be a felt need 
Table 3 Research during training

How often did you record standard outcome measures of the RDs during training?

\begin{tabular}{|c|c|}
\hline Always & $4(7.55 \%)$ \\
\hline Often & $34(64.15 \%)$ \\
\hline Seldom & $13(24.53 \%)$ \\
\hline Never & $1(1.89 \%)$ \\
\hline Other & $1(1.89 \%)$ \\
\hline \multicolumn{2}{|l|}{ Kind of thesis during training } \\
\hline Clinical cohort/case control study & $17(32.1 \%)$ \\
\hline Retrospective data collection & $40(75.5 \%)$ \\
\hline Clinical trial & $14(26.4 \%)$ \\
\hline Basic immunology/ laboratory based & $14(26.4 \%)$ \\
\hline On a scale of $0-10$, to what extent your thesis changed your patient care perspective (median IQR) & $5.5(5-8)$ \\
\hline \multicolumn{2}{|l|}{ Familiarity with publication ethics } \\
\hline Attended a structured course & $19(35.9 \%)$ \\
\hline Confident based on self-reading & $17(32.1 \%)$ \\
\hline Familiar but not confident & $24(45.3 \%)$ \\
\hline Neither familiar nor confident & $3(5.7 \%)$ \\
\hline \multicolumn{2}{|l|}{ Familiarity with statistical analysis } \\
\hline Attended a structured course & $17(32.1 \%)$ \\
\hline Confident based on self-reading & $11(20.8 \%)$ \\
\hline Familiar but not confident & $28(52.8 \%)$ \\
\hline Neither familiar nor confident & $6(11.3 \%)$ \\
\hline On a scale of $0-10$, how adequate is the time allotted for research (median IQR) & $6(2-6)$ \\
\hline On a scale of $01-10$, how compulsory should research be on training curriculum? (Median IQR) & $10(6-10)$ \\
\hline \multicolumn{2}{|l|}{ What could have boosted research initiative during training? } \\
\hline Exclusive time apart from regular clinical/ academic duties & $42(79.25 \%)$ \\
\hline Relevant topics & $19(35.85 \%)$ \\
\hline Dedicated funds & $32(60.38 \%)$ \\
\hline Support staff & $30(56.60 \%)$ \\
\hline Better academic career opportunities & $21(39.62 \%)$ \\
\hline Not applicable & $1(1.89 \%)$ \\
\hline
\end{tabular}

for improvement in training in specific areas such as laboratory, radiology and ancillary care and to overhaul assessment system by including objective evaluation.

A significant proportion of respondents felt that more emphasis should be laid to develop a structured and robust curriculum to impart the right mix of theoretical knowledge and practical clinical skills using standard and innovative teaching methods [8], for example, small interactive group sessions, flipped classroom learning, the use of patient educators/partners, team-based learning, peer-assisted learning, e-learning or computer-assisted learning as a useful adjunct [9].

Our study highlights the need to increase the trainee exposure in practical skills such as laboratory, immunology techniques and radiology methods which should be made compulsory in rheumatology training. Radiology experience is a vital aspect of the quality of rheumatology training and is considered a valuable training strategy for UK trainees
[10]. 'Shape of training' reform in 2019 has transformed the training in the UK [11]. Generalistic post-graduate training followed by speciality training ensures adequate exposure in general internal medicine followed by specialist clinical rheumatology. UK training system allows rheumatology trainees to embark on an academic route to pursue academic careers (reference). Likewise, structured academic rheumatology training in India would help encouraging trainees to explore and enjoy academic rheumatology.

Patient exposure and rheumatic disease management expertise of the trainees are really well considering the huge footfall of patients in rheumatology departments of most centres. Limited training highlighted in paediatric rheumatology and soft tissue rheumatism needs to be addressed to improve the quality of teaching programs in India. Trainees face similar problems in USA, due to lack of paediatric rheumatology training in centres other than the hospitals consisting paediatric rheumatologists which are few and far 
Table 4 Assessment system and CME

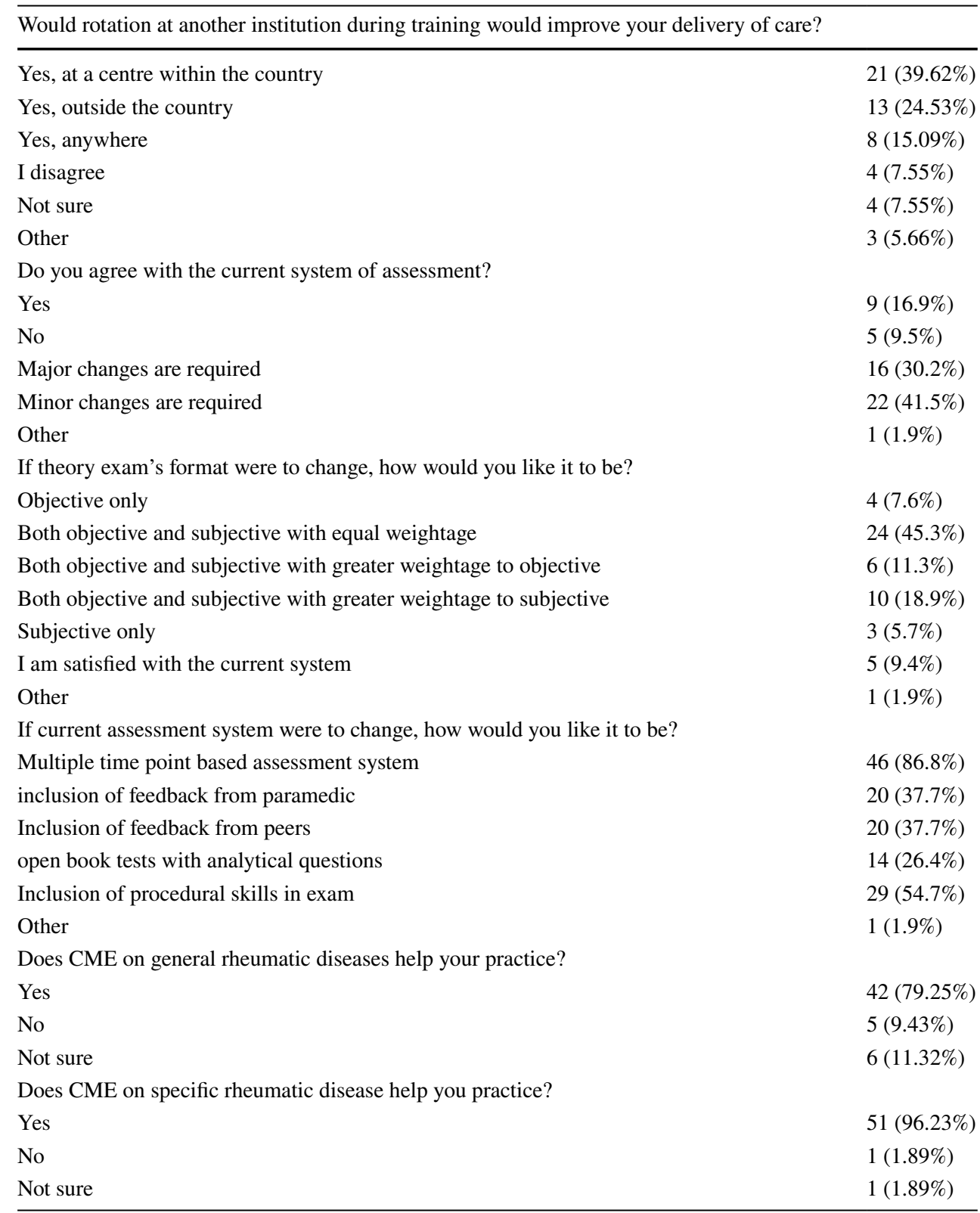

between. The lack of exposure to paediatric rheumatology during residency can impede a clinicians' ability to diagnose life-threatening RDs in children because many will present to non-specialists that do not have the specialist training in paediatric rheumatology. With a distinct pathophysiology and management approach required, paediatric rheumatology deserves a separate training programme. Well-established paediatric rheumatology training programme in the UK ensures that trainees receive high quality, goal-oriented training with an interface for paediatric rheumatology to allow a smooth transition to adult rheumatology [12]. Thus, it should be a fundamental requirement to gain basic adult and paediatric RDs knowledge and competencies in global undergraduate medical education [13].
Our study shows that the trainees are highly skilled in administering intra-articular injections but lag behind in invasive procedural skills such as skin biopsy, nerve biopsy, muscle biopsy, kidney biopsy and sonographically guided procedure. Most biopsies can be conducted with the help of allied specialities; consequently, it is not a limitation in patient care. On the other hand, ultrasound (US) guided procedures are of therapeutic and diagnostic importance in rheumatology. US-guided procedures are used for common interventions such as aspiration of synovial fluid, USguided injections and tissue biopsies [14]. Thus, the training of sonographically guided procedures is a merit of the Indian rheumatology training programmes, and their inclusion may be considered by programmes around the world $[15,16]$. 
It was worrisome to note that a significant proportion of (two-thirds) practitioners were not confident to address cardiovascular risk assessment, bone health, physiotherapy, rehabilitation, vaccination, mental health issues and patient counselling in patients with RDs. Mental health and community training form an essential part of the curriculum, especially in paediatric rheumatology [17]. Mental health disorders are prevalent in $20 \%$ of the Indian population and around $48 \%$ in RDs $[18,19]$. Thus, it seems imperative to include these in the Indian training system too. However, the trainees' perception was that allied service personnel such as nurses, physiotherapists and counsellors are better suited to deal with mental health issues in patients with RDs because they receive formal training include workshops and lectures. Trainees were also hesitant to deal with social, cultural and gender-specific issues [20]. In a resource-constrained setting in the developing world may limit a patient's ability to bear healthcare expenses incurred by ancillary services, the burden of collateral advice frequently falls upon the physician. Moreover, to deliver wholesome care, formal training of the basic tenets of ancillary services is advisable.

Internship and fellowship programs may invest heavily in teaching skills for conducting research and clinical skills, but they forget to focus enough on how to translate scientific evidence into practice, programs and policy [21]. Training for generic skills such as communication, leadership and management is deemed essential in the UK curriculum in addition to clinical skills [3]. These generic skills lay the foundation for effectiveness in delivering guidance for patient care, influencing health policies and patient communication.

A majority of trainees were actively involved in clinical research, which included writing case reports and cohort studies. However, review articles, meta-analysis, retrospective studies, lab-based studies and clinical trials were the forms of research that trainees were less familiar with and required to be promoted for a more holistic approach to academic learning. Submission of dissertation/thesis project is a mandatory requirement for a postgraduate qualification (DM/DNB) in rheumatology in India [3]. Most respondents felt that their research work improved clinical care delivery in unique ways. However, the respondents' familiarity with publication ethics, statistical methods of research and research methodologies was lacking. Although only a minority of rheumatology trainees eventually choose to be academicians, it is imperative to impart them sufficient levels of knowledge regarding the ethics and don'ts in publishing practices. The ongoing Covid-19 pandemic has led to a surge in predatory journals and misinformation and identifying valid and ethical research is mandatory to synthesize valid conclusions amid the large volume of published literature. The bulk of trainees in our study recommended that epidemiology and statistics specifically be a part of the training curriculum in rheumatology to further their research skills.
To advance the research initiatives during training, the curriculum should include exclusive time apart from regular clinical and academic duties, dedicate funds, support from staff and procurement of better academic opportunities for the availability of relevant research topics.

Rheumatology and immunology are deeply entwined, and an understanding of the immune basis of RD is the stepping stone towards hypothesis formulation, bench to bedside research and precision medicine. A sizable proportion of respondents were not trained in basic immunology laboratory techniques, bringing this deficit to the fore. Including certain forms of basic rheumatology training at the undergraduate level may help with a holistic transition to a physician [8]. In a time when biologics and targeted synthetic disease-modifying anti-rheumatic drugs (DMARDs) are blooming, it is essential to understand the pathogenic origins to gather the probability of clinical success and expected adverse effects in the patients.

Notably, a majority of trainees participating in our study were dissatisfied with the current system of assessment employed in rheumatology training. The suggested changes included multiple time point-based assessment systems (monthly or fortnightly), the inclusion of procedural skills in the exam, and inclusion of feedback from paramedic staff and peers, combining objective and subjective assessment, collaborative programs at other institutes within the country and regular CME workshops on general rheumatology practice and specific RDs. These will enhance the level of rheumatology training in India and help them in their clinical practice [22]. The arrangement of CME workshops should be a beneficial addition in rheumatology training as respondents (96\%) in our survey unanimously agreed that CMEs on specific RDs helped in their practice. CME workshops result in a small but impactful improvement in professional practice and patient outcomes. However, interactive education, in combination with didactic teaching in CME workshops, is a developing but highly effective strategy for clinical practice enhancement [23]. Semi-virtual models of teaching and use of social media in medicine are on the rise and maybe put to greater use in the near future $[24,25]$. A study conducted on the importance of clinical assessments for rheumatology training states that formative assessments are crucial through the feedback from both trainees and trainers [26]-28 It is a great motivator for improvement in any clinical skill deficiencies. The study also highlighted the inclusion of procedural skills such as imaging and laboratory stations which correspond to the suggestions from the trainees in our study. Remote assessment of rheumatic diseases has gained a greater foothold during the pandemic and maybe another terrain to explore in the near future [29, 30].

Interdepartmental meetings, especially with radiologist and pathologists, can provide valuable insights into key aspects of diagnostics. This is an often ignored domain at 
some centres and deserves greater attention for proficiency in patient care [9]. Team-based and computer-assisted interactive learning models can be useful adjuncts to enhance the efficiency of the training programmes. Prior to the implementation of any curriculum changes, it is essential to consider how the intended outcomes will be assessed with respect to both immediate and longer-term impact. Rheumatology programmes should aim to train students into assuming a larger role of an expert and deal with complex scenarios by deconstructing the symptom, sign and investigation cluster with diligence and enthusiasm with the vision to serve as good role-models [31]. Education grants and scholarships may be a useful model in rheumatology training [32]. We believe that this grant may serve as a model for other medical societies that want to promote education scholarship and leadership within their specialities. Harmonising training programs across countries and centres can foster the development of a network involving competent specialist centres [12,33]. This will facilitate collaborative research and training opportunities. Last, transitional care is an unmet challenge all over the world. We hope that despite the scarcity of the paediatric rheumatologists, incorporation of specific aspects of adolescent problems and mental health can improve the delivery of care and transition to adulthood [34]. Indian Rheumatology Association (IRA) plays an important role in overseeing and influencing policy change to facilitate structured rheumatology training in India.

We acknowledge the limitations inherent to the design of a survey-based study, including non-response and recall bias. A limited number of responses is another disadvantage as subgroup analysis would be rendered unfeasible, despite numerous reminders spread over 5 months. However, since this study was conducted in the pandemic period, remote reminders were the only feasible means to request a response. Nevertheless, our study is the first formal assessment of perspectives of rheumatology trainees regarding their curriculum. We hope that this pilot study would help in the identification of the curricular deficiencies in rheumatology training in India towards efforts to improve superspeciality training.

\section{Conclusion}

Although rheumatology in India has grown in recent years, there is an unmet need to cater for the growing population. Collaborative efforts to improve training, education and research can facilitate the future growth of rheumatology in India. Unwavering efforts of dialogue and negotiations with policymakers would help to achieve this goal.

Supplementary Information The online version contains supplementary material available at https://doi.org/10.1007/s00296-021-04962-9.
Acknowledgements None.

Author's contribution Conceptualization: MS, HK, DPM, RK, AN, VA, LG. Data curation: MS, HK, DPM, RK, AN, VA, LG. Formal analysis: MS, HK, LG. Funding acquisition: N/A. Investigation: MS, HK, DPM, RK, AN, VA, LG. Methodology: MS, LG. Software: N/A. Validation: N/A. Visualization: MS, DPM, RK, LG. Writing—original draft: MS, HK, RK. Writing—review \& editing: AN, VA, LG.

Funding None.

Code availability Not applicable.

\section{Declarations}

Conflicts of interest None.

Ethics clearance Not applicable.

Consent to participate Not applicable.

Consent to publication Yes.

\section{References}

1. Chopra A (2013) The COPCORD world of musculoskeletal pain and arthritis. Rheumatology 52:1925-1928. https://doi.org/10. 1093/rheumatology/ket222

2. Misra DP, Agarwal V, Negi VS (2016) Rheumatology in India: a bird's eye view on organization, epidemiology, training programs and publications. J Korean Med Sci 31:1013-1019. https://doi. org/10.3346/jkms.2016.31.7.1013

3. Das P, Moorthy A, Dharmanand B (2018) Rheumatology training in India compared to the United Kingdom, Indian. J Rheumatol 13:113-116. https://doi.org/10.4103/injr.injr_78_17

4. Misra DP, Sharma A, Agarwal V (2018) Rheumatology science and practice in India. Rheumatol Int 38:1587-1600. https://doi. org/10.1007/S00296-018-4111-4

5. Dua $\mathrm{AB}$ et al (2020) Challenges, collaboration, and innovation in rheumatology education during the COVID-19 pandemic: leveraging new ways to teach. Clin Rheumatol 39:1. https://doi.org/10. 1007/S10067-020-05449-X

6. Zamanzadeh V, Ghahramanian A, Rassouli M, Abbaszadeh A, Alavi-Majd H, Nikanfar A-R (2015) Design and Implementation Content Validity Study: development of an instrument for measuring patient-centered communication. J Caring Sci 4:165-178. https://doi.org/10.15171/jcs.2015.017

7. Gaur PS, Zimba O, Agarwal V, Gupta L (2020) Reporting survey based studies-a primer for authors. J Korean Med Sci 35:45. https://doi.org/10.3346/jkms.2020.35.e398

8. Pangtey G, Gulati S, Maheshwari A (2018) Improving knowledge and awareness regarding rheumatology at the undergraduate level in Indian medical colleges. Indian J Rheumatol 13:217. https:// doi.org/10.4103/0973-3698.244176

9. Al Maini M et al (2015) The global challenges and opportunities in the practice of rheumatology: white paper by the World Forum on rheumatic and musculoskeletal diseases. Clin Rheumatol 34:819-829. https://doi.org/10.1007/s10067-014-2841-6

10. Dubey SG, Roberts C, Adebajo AO, Snaith ML (2004) Rheumatology training in the United Kingdom: the trainees' perspective. Rheumatology (Oxford) 43:896-900. https://doi.org/10.1093/ rheumatology/keh192 
11. Rimmer A (2019) Training the new generalists. BMJ 366:15042. https://doi.org/10.1136/bmj.15042

12. Foster HE et al (2018) Educational initiatives and training for paediatric rheumatology in Europe. Pediatr Rheumatol 16:77. https:// doi.org/10.1186/s12969-018-0289-y

13. Al Maini M et al (2020) A global perspective on the challenges and opportunities in learning about rheumatic and musculoskeletal diseases in undergraduate medical education. Clin Rheumatol 39:627-642. https://doi.org/10.1007/s10067-019-04544-y

14. Koski JM, Hammer HB (2012) Ultrasound-guided procedures: techniques and usefulness in controlling inflammation and disease progression. Rheumatology (Oxford) 51:vii31-vii35. https://doi. org/10.1093/rheumatology/kes331

15. Taggart A et al (2006) Musculoskeletal ultrasound training in rheumatology: the Belfast experience. Rheumatology 45:102-105. https://doi.org/10.1093/rheumatology/kei162

16. Wu M et al (2021) Musculoskeletal ultrasound imaging training, use, and knowledge among rheumatologists in China. Clin Rheumatol 40:321-330. https://doi.org/10.1007/s10067-020-05175-4

17. Davis AM, Rubinstein TB, Rodriguez M, Knight AM (2017) Mental health care for youth with rheumatologic diseases-bridging the gap. Pediatr Rheumatol 15:85. https://doi.org/10.1186/ s12969-017-0214-9

18. Math SB, Srinivasaraju R (2010) Indian Psychiatric epidemiological studies: learning from the past, Indian. J Psychiatry 52:S95S103. https://doi.org/10.4103/0019-5545.69220

19. Matcham F, Rayner L, Steer S, Hotopf M (2013) The prevalence of depression in rheumatoid arthritis: a systematic review and meta-analysis. Rheumatology (Oxford) 52:2136-2148. https://doi. org/10.1093/rheumatology/ket169

20. Balakrishnan A, Mehta P, Gupta L (2020) Pregnancy counseling in rheumatic diseases: where science meets the steps. Indian J Rheumatol. https://doi.org/10.4103/injr.injr_79_20

21. Shlafer RJ, McRee A-L, Gower AL, Bearinger LH (2016) Better communication for better public health: perspectives from an interdisciplinary training program. Health Promot Pract 17:165168. https://doi.org/10.1177/1524839915627453

22. Hassell A (2017) Higher medical training in Rheumatology in the UK: any lessons worth learning? Mediterr J Rheumatol 28:171173. https://doi.org/10.31138/mjr.28.4.171

23. Forsetlund $\mathrm{L}$ et al (2009) Continuing education meetings and workshops: effects on professional practice and health care outcomes. Cochrane database Syst Rev 2009:CD003030. https://doi. org/10.1002/14651858.CD003030.pub2

24. Sinha M, Agarwal V, Gupta L (2020) Human touch in digital education—a solution. Clin Rheumatol 39:3897-3898. https://doi. org/10.1007/s10067-020-05448-y
25. A. S, Z. O, and G. AY, (2020) Moving towards online rheumatology education in the era of COVID-19. Clin Rheumatol 39:32153222. https://doi.org/10.1007/S10067-020-05405-9

26. Alunno A, Najm A, Sivera F, Haines C, Falzon L, Ramiro S (2020) Assessment of competences in rheumatology training: results of a systematic literature review to inform EULAR points to consider. RMD Open 6:e001330. https://doi.org/10.1136/rmdop en-2020-001330

27. Ahmed S, Gupta L (2020) Perception about social media use by rheumatology journals: survey among the attendees of IRACON, Indian. J Rheumatol 15:171-174. https://doi.org/10.4103/injr.injr_ $15 \_20$

28. Gupta L, Agarwal V, Misra R (2020) Changing the way scientific meetings are conducted—results from a survey conducted among attendees of a technology-modeled rheumatology meeting. JCR. https://doi.org/10.1097/RHU.0000000000001680

29. Ahmed S, Gupta L (2020) Social media for medical journals. Cent Asian J Med Hypotheses Ethics 1:26-32. https://doi.org/10. 47316/cajmhe.2020.1.1.04

30. Naveen R, Sundaram TG, Agarwal V, Gupta L (2021) Teleconsultation experience with the idiopathic inflammatory myopathies: a prospective observational cohort study during the COVID-19 pandemic. Rheumatol Int 41:67-76. https://doi.org/10.1007/ s00296-020-04737-8

31. Wilson AS et al (2006) Development of an interactive learning tool for teaching rheumatology-a simulated clinical case studies program. Rheumatology 45:1158-1161. https://doi.org/10.1093/ rheumatology/kel077

32. Berman JR et al (2016) Rheumatology research foundation clinician scholar educator award: fifteen years promoting rheumatology educators and education. Arthritis Care Res (Hoboken) 68:1591-1597. https://doi.org/10.1002/acr.22858

33. Barbour V, Kleinert S, Wager E, Yentis S (2009). Guidelines for retracting articles. https://doi.org/10.24318/cope.2019.1.4

34. J. E. McDonagh, T. R. Southwood, K. L. Shaw, and on behalf of the B. P. R. Group (2004) Unmet education and training needs of rheumatology health professionals in adolescent health and transitional care. Rheumatology 43:737-743. https://doi.org/10.1093/ rheumatology/keh163

Publisher's Note Springer Nature remains neutral with regard to jurisdictional claims in published maps and institutional affiliations. 